\title{
Bending Poisson Effect in Two-Dimensional Crystals
}

\author{
Xiaofei Liu, ${ }^{1}$ Douxing Pan, ${ }^{2}$ Yuanzhou Hong, ${ }^{2}$ and Wanlin Guo ${ }^{1, *}$ \\ ${ }^{1}$ State Key Laboratory of Mechanics and Control for Mechanical Structures and Key Laboratory for Intelligent Nano Materials and \\ Devices (MOE), Nanjing University of Aeronautics and Astronautics, Nanjing 210016, China \\ ${ }^{2}$ Institute of Mechanics, Chinese Academy of Science, Beijing 100190, China
}

(Received 7 August 2013; published 21 May 2014)

\begin{abstract}
As the Poisson effect formulates, lateral strains in a material can be caused by a uniaxial stress in the perpendicular direction, but no net lateral strain should be induced in a thin homogeneous elastic plate subjected to a pure bending load. Here, we demonstrated by ab initio simulations that significant exotic lateral strains can be induced by pure bending in two-dimensional crystals, in which the lateral components of chemical bonds can respond to bending curvature directly. The bending Poisson ratio, defined as the ratio of lateral strain to the curvature, is a function of curvature depending on chemical constitution, bonding structure, and atomic interaction of the crystal, and is anisotropic.
\end{abstract}

PACS numbers: 62.20.- $\mathrm{x}, 46.70 .-\mathrm{p}, 61.46 .-\mathrm{w}, 81.07 .-\mathrm{b}$

The Poisson effect as schematically shown in Fig. 1(a) is an essential behavior of material. When subjected to a uniaxial tension or compression load, a solid material usually shrinks or expands laterally due to its resistance to alter in volume [1,2]. The Poisson ratio, namely the negative ratio of the induced lateral strain to the strain parallel to the uniaxial stress, is found to be dependent on the atomic packing density and valance electron density of the material $[3,4]$. Since the 1980s, it has been reported that materials of reentrant structures can possess an exotic negative Poisson ratio [5-8]. In practical situations, materials are always in complicated stress states. In a thin homogeneous elastic plate subjected to pure bending load, tensile and compressive stresses will antisymmetrically distribute in the two sides of the middle neutral plane, as illustrated in Fig. 1(b). In this perspective, the lateral contractive and expansive strains aroused owing to the Poisson effect in the plate are aligned antisymmetrically, and should counteract each other, leading to a zero net strain in the lateral direction. In this Letter, we reveal by comprehensive $a b$ initio simulations that single-layered two-dimensional (2D) crystals, such as transition metal dichalcogenides (TMDs), fluorinated graphene, and hexagonal boron nitride (h-BN), and even pristine h-BN, can experience exotic lateral strains under pure bending, termed bending Poisson effect here. The underlying physical mechanisms are systematically studied.

To mimic the bending deformation in single-layered materials, the single-layered sheets are rolled into the corresponding tubes with the tube radii assigned as the radii of curvature. The strategy has been widely adopted to investigate the bending rigidity of graphene $[9,10]$ and has a specific advantage here in that the curvature of bending and the curvature-induced lateral strain are homogeneous around the circumference. The geometry relaxations are carried out using density functional theory (DFT) as implemented in the VASP code $[11,12]$, with the projector augmented wave method for the core region and the generalized gradient approximation of the Perdew-Burke-Ernzerhof functional for the exchangecorrelation potential [13-15]. High accuracy settings are adopted in simulations [16].

For briefness of discussion, the crystal orientations parallel and perpendicular to the moment of force are assigned as the lateral and the bending directions, respectively. Based on DFT results, the lateral strain is calculated as $\varepsilon_{L}=\left(c_{b}-c_{0}\right) / c_{0}$, where $c_{0}$ and $c_{b}$ are the lateral lattice constants in the strain-free and the bent states. Since the lateral strain may be constrained by boundary conditions in a practical bending deformation, an equivalent lateral stress can be induced by bending and written as $\sigma_{L}=Y \varepsilon_{L}$, where $Y$ is the in-plane Young's modulus. A power law $\varepsilon_{L}=C k^{\lambda}$ is suitable to describe the lateral strains as a function of curvature $k$, with the coefficient $C$ and exponent $\lambda$ determined by fitting the DFT results. Following the $\varepsilon_{L}-k$ relation, the bending Poisson ratio $\mu$, defined as the ratio of the lateral (a)

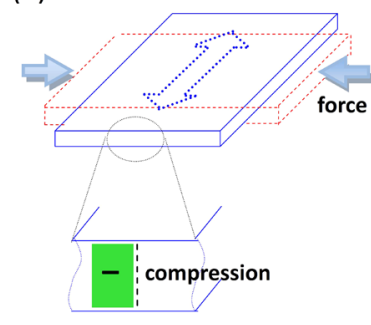

(b)

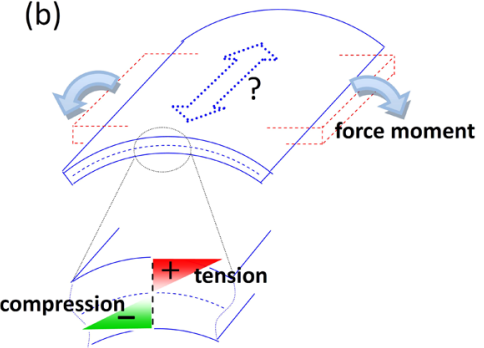

FIG. 1 (color online). (a) Poisson effect in a homogeneous elastic plate under a uniaxial load. (b) A homogeneous elastic plate under a pure bending load. The plates with and without mechanical load are depicted by the red dashed and blue solid lines, respectively. The blue dashed arrows represent the deformation-induced lateral strain. The lower panels illustrate the stress conditions at the cross sections. 


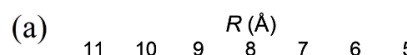

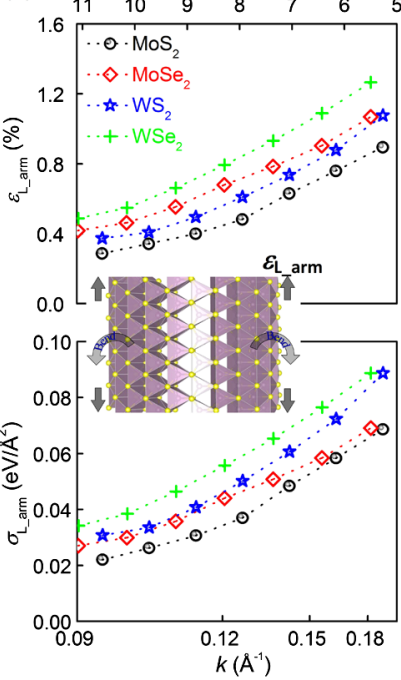

(b) $R(\AA)$

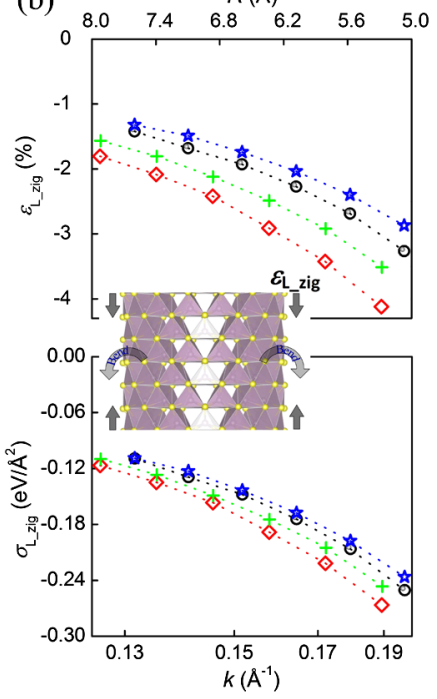

FIG. 2 (color online). Bending Poisson effect in single-layered TMDs. (a),(b) Bending induced lateral strain and equivalent lateral stress as a function of curvature for bending along the armchair (a) and the zigzag (b) directions in single-layered $\mathrm{MoS}_{2}$, $\mathrm{MoSe}_{2}, \mathrm{WS}_{2}$, and $\mathrm{WSe}_{2}$.

strain to the curvature, can be explicitly expressed as $\mu=C k^{\lambda-1}$. As $\varepsilon_{L}$ is a dimensionless quantity, it is more precise to rewrite the power law as $\varepsilon_{L}=C k^{\lambda}=c(h k)^{\lambda}$, where $h$ is the thickness of the sheet. Under the same bending curvature, thicker crystal has a larger maximum of bending strain and higher elastic energy according to the conventional plate theory. Then it is reasonable to assume that $\varepsilon_{L}$ can be in positive proportion to the thickness, when all other parameters are fixed. The constant $c$ shall reflect the influence by other factors of the material.

We first focus on finite-thickness 2D crystals, namely, single-layered materials with more than one chemically bonded atomic layer in thickness, as they stand for most common layered systems [17-20]. Without loss of generality, the bending Poisson effect in the single layer of TMD is systematically investigated as a prototype. Based on the $\varepsilon_{L^{-}} k$ and $\sigma_{L^{-}} k$ relations illustrated in Fig. 2 as well as the fitted coefficient $C$ and exponent $\lambda$ of the power law in Table I, four typical features of the bending Poisson effect in single-layered TMDs are observed.

(1) The bending Poisson ratio is a function of curvature. As the fitted exponents $\lambda$ in the power law for TMD bent along the zigzag direction is close to being 2 , the lateral strain $\varepsilon_{\text {Lzig }}$ follows a quadratic scaling with the curvature, while the bending Poisson ratio is linearly scaling to the curvature, in stark contrast to the constant Poisson ratio. More complicatedly, when being bent along the armchair direction, the exponent $\lambda$ can vary from 1.435 to 1.891 for the four TMDs.

(2) The bending Poisson effect is highly anisotropic. The lateral lattice expands when bent along the armchair direction, but shrinks when bent along the zigzag direction. What is more, the magnitude of the bending Poisson ratio at fixed curvature is much higher for bending along the zigzag direction (Table I). When the bending is along an arbitrary chiral direction, the bending-induced lateral strain should vary continuously between the armchair and zigzag cases.

(3) The bending-induced lateral strain can be significant. With the radius of curvature down to $\sim 5.5 \AA, \varepsilon_{L z i g}$ in $\mathrm{MoSe}_{2}$ is up to $-4.1 \%$, while $\varepsilon_{L \text { arm }}$ in $\mathrm{WSe}_{2}$ is up to $1.3 \%$. For the largest radii of curvature considered in the ab initio simulations, $\varepsilon_{\text {Lzig }}$ in $\mathrm{MoSe}_{2}(7.9 \AA$ in radius of curvature) and $\varepsilon_{L \text { arm }}$ in $\mathrm{WSe}_{2}$ (11.0 $\AA$ in radius of curvature) can still have a value of $-1.8 \%$ and $0.5 \%$, respectively. Based on the power law approximation, even in bent $\mathrm{MoSe}_{2}$ with a much larger radius of curvature of $30 \AA, \varepsilon_{\text {Lzig }}$ is estimated to be $-0.1 \%$, easy to be detected experimentally.

(4) Chemical constituents of the single-layered TMD have influences on the bending Poisson effect. While $\varepsilon_{L \text { arm }}$ is larger with heavier chalcogen and heavier metal atoms, $\varepsilon_{\text {Lzig }}$ is larger with heavier chalcogen but lighter metal atoms. The more significant bending Poisson effect in TMD with heavier chalcogen is understandable as they have larger thicknesses.

The responses of chemical bonds in single-layered $\mathrm{MoS}_{2}$ to curvature are shown in Figs. 3(b), 3(c), 3(e), and 3(f). The bonds, with their major components aligned along the bending and lateral directions, are denoted as $d_{1}$ and $d_{2}$,

TABLE I. The fitted coefficients $C, c$, and $\lambda$ in the power law approximation for bending-induced lateral strains in the $2 \mathrm{D}$ crystals. The first row illustrates the thicknesses of the strain-free 2D crystals. The last row presents the in-plane Young's modulus. The straincurvature and the bending Poisson ratio-curvature relations are described as $\varepsilon_{L}=C k^{\lambda}=c(h k)^{\lambda}$ and $\mu=C k^{\lambda-1}$, respectively.

\begin{tabular}{lccccccccc}
\hline \hline 2D crystals & & $\mathrm{MoS}_{2}$ & $\mathrm{MoSe}_{2}$ & $\mathrm{WS}_{2}$ & $\mathrm{WSe}_{2}$ & $\mathrm{~F}-\mathrm{GR}$ & $\mathrm{F}-\mathrm{BN}$ & Graphene & h-BN \\
\hline$h / \AA$ & & 3.13 & 3.34 & 3.14 & 3.35 & 3.25 & 3.30 & $\ldots$ & $\ldots$ \\
Armchair & $C / \AA^{\lambda}$ & 0.206 & 0.133 & 0.173 & 0.173 & 0.674 & 0.494 & $\ldots$ & $\ldots$ \\
& $\lambda$ & 1.811 & 1.435 & 1.633 & 1.480 & 1.891 & 1.888 & $\ldots$ & $\ldots$ \\
& $c$ & 0.026 & 0.024 & 0.027 & 0.029 & 0.073 & 0.052 & $\ldots$ & $\ldots$ \\
Zigzag & $C / \AA^{\lambda}$ & -0.855 & -1.215 & -0.656 & -0.934 & $\ldots$ & -0.155 & $\ldots$ & -0.038 \\
& $\lambda$ & 2.011 & 2.028 & 1.926 & 1.971 & $\ldots$ & 1.427 & $\ldots$ & 2.254 \\
& $c$ & -0.086 & -0.105 & -0.072 & -0.086 & $\ldots$ & -0.028 & $\ldots$ & $\ldots$ \\
$Y\left(\mathrm{eV}^{2}\right)$ & & 7.7 & 6.8 & 8.2 & 7.0 & 13.1 & 10.3 & 20.4 & 17.3 \\
\hline \hline
\end{tabular}


respectively [Figs. 3(a) and 3(d)]. When bent along the armchair direction, the bonds elongate in the tensile outer side $\left(d_{1 \text { out }}, d_{2 \text { out }}, d_{2^{\prime} \text { out }}\right)$ while shorten in the compressive inner side $\left(d_{2 \text { in }}, d_{2 \text { in }}, d_{2 \text { in }^{\prime}}\right)$, as expected by the conventional plate theory (note $d_{2}$ has a minor component along the bending direction). Although the elongation of $d_{2 \text { out }}$ is almost equal in magnitude to the shrinkage of $d_{2 \text { in }}$, the shrinkage in $d_{1 \text { in }}$ is much less than the elongation in $d_{1 \text { out }}$, going against the conventional theory. Meanwhile, the S-Mo-S angle $\theta_{\text {out }}$ between the bonds $d_{2 \text { out }}$ and $d_{2 \text { out }}$ slightly decreases with curvature, while the S-Mo-S angle $\theta_{\text {in }}$ between the bonds $d_{2 \text { in }}$ and $d_{2 \text { in }^{\prime}}$ remarkably increases. As the lateral lattice constant can be written as $c_{L}=$ $2 d_{2 \text { in }} \sin \left(\theta_{\text {in }} / 2\right)$ or $c_{L}=2 d_{2 \text { out }} \sin \left(\theta_{\text {out }} / 2\right)$, such joint
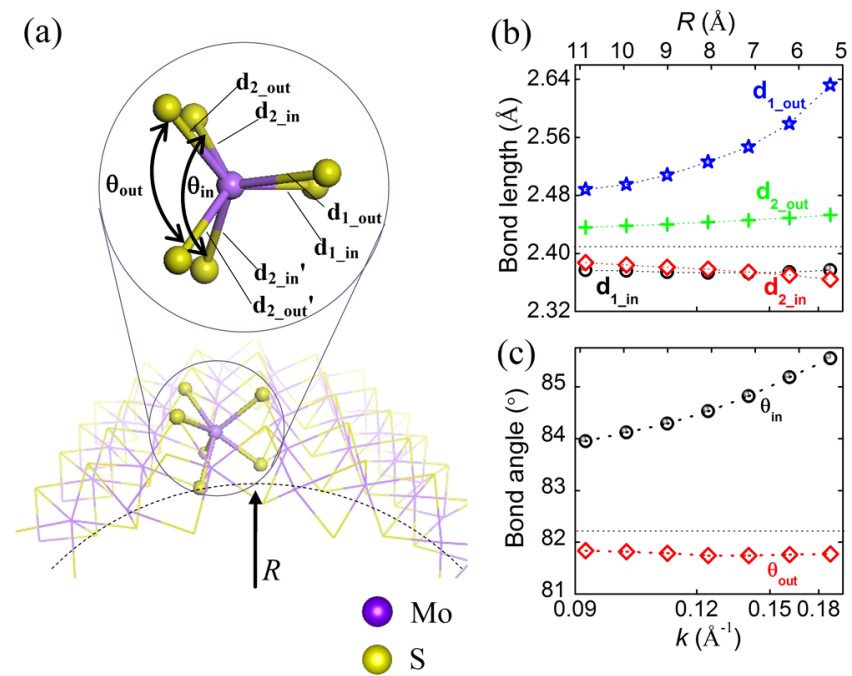

(d)
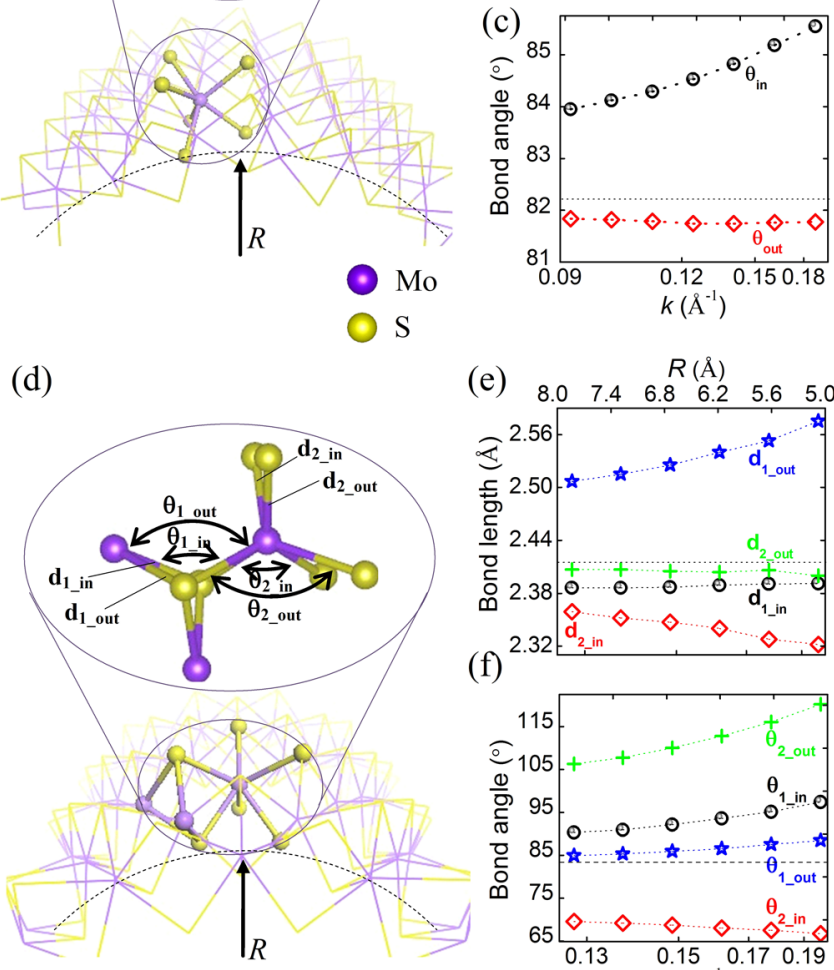

(e)
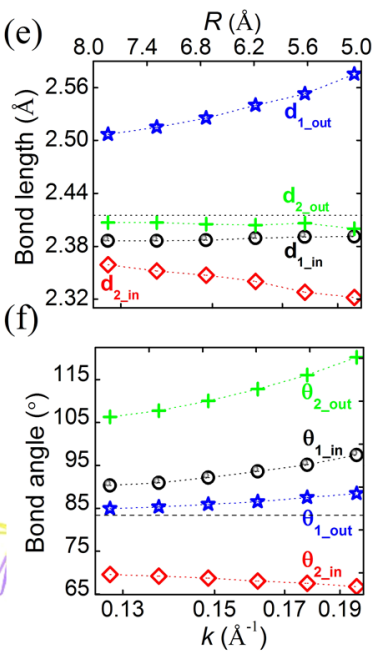

FIG. 3 (color online). Responses of chemical bonds in the single-layered $\mathrm{MoS}_{2}$ under pure bending. (a) A single-layered $\mathrm{MoS}_{2}$ bent along the armchair direction. (b), (c) The bond lengths and bond angles as a function of curvature. The bond lengths and bond angles are labeled in (a). Corresponding results for the bending along the zigzag direction are presented in (d), (e), and (f). The dashed lines in (b), (c), (e), and (f) show the bond length or bond angle in strain-free $\mathrm{MoS}_{2}$. responses of bond lengths and angles result in the overall expansion of lateral lattice. When bent along the zigzag direction, $d_{1 \text { out }}$ elongates remarkably but $d_{1 \text { in }}$ slightly shortens, similar to the armchair case. Both $d_{2 \text { in }}$ and $d_{2 \text { out }}$ perpendicular to the direction of bending are shortened, contradicting the conventional theory. The asymmetry between the elongation in the tensile side and shrinkage in the compressive side can be, at least partially, explained by charge transfer along the thickness direction, as the Mo atoms provide more electrons to the $\mathrm{S}$ atoms at the compressive side. (see Fig. S1 in the Supplemental Material for the deformation electron density [21]).

The bending Poisson effect is also identified in other types of finite-thickness 2D crystals, such as fluorinated graphene and fluorinated hexagonal boron nitride (F-GR and F-BN) [22,23]. As shown in Figs. 4(a) and 4(b), the bending-induced lateral strains in the fluorinated atomic layers are significant, with the same order of magnitude and similar anisotropy as that in single-layered TMDs. The power law can well describe the $\varepsilon_{L a r m}-k$ relations in both F-GR and F-BN, with an exponent around 1.89. However, the $\varepsilon_{\text {Lzig }}-k$ relation is abnormal in the fluorinated atomic layers bent along the zigzag direction: $\varepsilon_{\text {Lzig }}$ in the F-GR converges to a constant value as the radius decreases to $6 \AA$,
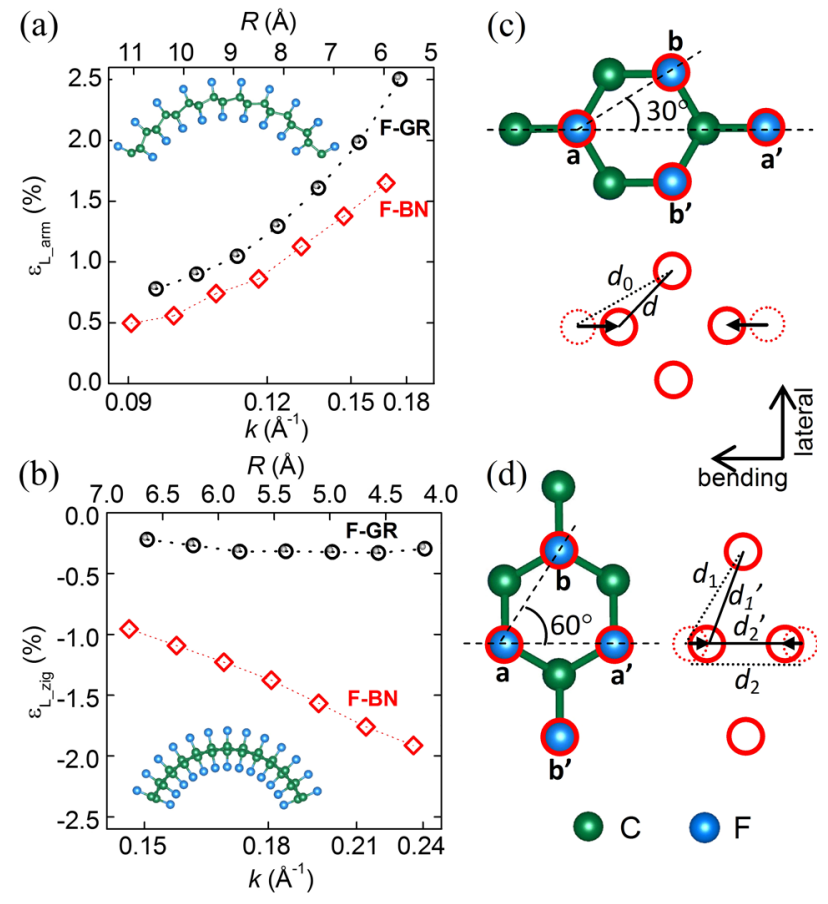

FIG. 4 (color online). Bending Poisson effect in single-layered fluorinated graphene and fluorinated h-BN. Bending induced lateral strain as a function of curvature for bending along the armchair (a) and the zigzag (b) directions. The change of the separation between neighboring $\mathrm{F}$ atoms in the compression side is illustrated for bending along (c) the armchair and (d) the zigzag directions. 
whereas in the F-BN the exponent $\lambda$ of the power law has a lowest value of 1.427 .

The C-F bonds in the F-GR are perpendicular to the in-plane directions and, thus, cannot respond to bending directly. Instead, both the in-plane $\mathrm{C}-\mathrm{C}$ and the perpendicular $\mathrm{C}-\mathrm{F}$ bonds are influenced indirectly through the Coulomb interaction between the highly electronegative surface fluorine atoms. For illustration, the interaction between the fluorine atoms on the compressive side is depicted in Figs. 4(c) and 4(d). When a F-GR is bent along the armchair direction, the distance between the nearest neighboring fluorine $a$ and $b$ is shortened remarkably as the vector $\boldsymbol{a} \boldsymbol{b}$ only cuts a $30^{\circ}$ angle with the bending direction. Then the fluorine atoms in the compressive side experience a strong interactive repulsive force, leading to the elongation in the C-C bonds along the lateral direction [24]. When bent along the zigzag direction, the distance between the nearest neighboring fluorine $a$ and $b$ is slightly shortened as the vector $\boldsymbol{a} \boldsymbol{b}$ cuts a $60^{\circ}$ angle with the direction of bending, but the distance between the nearest neighboring fluorine $a$ and $\boldsymbol{a}^{\prime}$ is shortened remarkably since the vector $\boldsymbol{a} \boldsymbol{a}^{\prime}$ is parallel to bending, resulting in strong repulsive forces merely along the bending direction. Then the compressive $\varepsilon_{\text {Lzig }}$ is aroused by the internal tensile stress along the bending direction due to the conventional Poisson effect. The above mentioned abnormal and smaller $\varepsilon_{\text {Lzig }}$ in F-GR is due to its lower conventional Poisson ratio than that of F-BN.

Nevertheless, the reduced dimensionality of a singlelayered material is not a sufficient condition for the generation of the bending Poisson effect. Though the conventional plate theory of bending is broken down in the monolayer graphene due to its ultrathin nature [25], we observe no obvious bending-induced lateral strain in graphene (see Fig. S2 of the Supplemental Material [21]), consistent with previous reports $[9,25]$. Regardless of the curvature, $\varepsilon_{L \text { arm }}$ is approximately zero even when the radius is down to $\sim 5.0 \AA$, while $\varepsilon_{L z i g}$ is always lower than $0.06 \%$ and rather random. In the monolayer h-BN, no lateral strain appears either when bent along the armchair direction. However, when bent along the zigzag direction, a tiny but evident lateral strain is observed, with a magnitude 1 order lower than that in TMDs. The power law still works for $\varepsilon_{\text {Lzig }}$ in h-BN with an exponent $\lambda$ higher than 2 (Table I). The essential difference between graphene and h-BN relies on the polarity of h-BN. The bond angles B-N-B are relatively flexible so that one of them can be distorted from $120^{\circ}$ to $116.35^{\circ}$ when the sheet is bent to a radius of $\sim 4.0 \AA$ along the zigzag direction (Fig. S2 in the Supplemental Material [21]). In contrast, the stiffer bond angles N-B-N remain nearly undistorted. The divergence between the two bond angles leads to an out-of-plane buckling and resultant shrinkage of the lateral lattice.

The mechanisms for the bending Poisson effect revealed above can be reproduced in other single-layered materials, such as gallium selenide, hydrogenated graphene [26], and hypothetical hexagonal aluminum nitride [27] (see Table SI of the Supplemental Material [21]). The common existence of the bending Poisson effect reflects a major difference between a 2D crystal and a bulk plate: the local lateral strain in a bulk plate is related to the local strain along the direction of bending due to the Poisson effect; in a finitethickness 2D crystal, the lateral strain responds to the curvature directly although the tensile and compressive features are still observed in its outer and inner sides; in a monolayer, such as h-BN without of tensile and compressive features, the lateral lattice constant reacts to bending as well.

With the insight into the basic constitutive relation of bending Poisson effect, we further incorporate the effect into the plate theory of large deflection [28]. In our revised theory [29], the strain-stress relation is modified to be

$$
\begin{aligned}
\sigma_{x} & =\frac{E}{1-\nu^{2}}\left(\varepsilon_{x}+\nu \varepsilon_{y}\right)-\sigma_{x l}, \\
\sigma_{y} & =\frac{E}{1-\nu^{2}}\left(\varepsilon_{y}+\nu \varepsilon_{x}\right)-\sigma_{y l}, \\
\sigma_{x y} & =G \varepsilon_{x y}, \\
\sigma_{x l} & =E \varepsilon_{x l}=E C_{y} k_{y}^{\lambda}, \\
\sigma_{y l} & =E \varepsilon_{y l}=E C_{x} k_{x}^{\lambda} .
\end{aligned}
$$

The lateral stress and strain induced by the curvature $k_{y}\left(k_{x}\right)$ due to the bending Poisson effect are denoted as $\sigma_{x l}\left(\sigma_{y l}\right)$ and $\varepsilon_{x l}\left(\varepsilon_{y l}\right)$, respectively. Defining a stress function $F$ as

$$
\frac{\partial^{2} F}{\partial x^{2}}=\sigma_{y}, \quad \frac{\partial^{2} F}{\partial y^{2}}=\sigma_{x}, \quad \frac{\partial^{2} F}{\partial x \partial y}=-\sigma_{x y},
$$

the equilibrium equation and deformation compatibility equation can be written, respectively, as

$$
\begin{aligned}
\frac{D}{h} \nabla^{4} w= & \frac{q}{h}+\frac{\partial^{2} F}{\partial y^{2}} \frac{\partial^{2} w}{\partial x^{2}}+\frac{\partial^{2} F}{\partial x^{2}} \frac{\partial^{2} w}{\partial y^{2}}-2 \frac{\partial^{2} F}{\partial x \partial y} \frac{\partial^{2} w}{\partial x \partial y}, \\
\nabla^{4} F & -\frac{\partial^{2}}{\partial y^{2}}\left[C_{y}\left(\frac{\partial^{2} w}{\partial x^{2}}\right)^{\lambda}-\nu C_{x}\left(\frac{\partial^{2} w}{\partial y^{2}}\right)^{\lambda}\right] \\
& -\frac{\partial^{2}}{\partial x^{2}}\left[C_{x}\left(\frac{\partial^{2} w}{\partial y^{2}}\right)^{\lambda}-\nu C_{y}\left(\frac{\partial^{2} w}{\partial x^{2}}\right)^{\lambda}\right] \\
= & E\left[\left(\frac{\partial^{2} w}{\partial x \partial y}\right)^{2}-\frac{\partial^{2} w}{\partial x^{2}} \frac{\partial^{2} w}{\partial y^{2}}\right]
\end{aligned}
$$

where $D$ is the bending stiffness and $q$ is the external force. Equations (3) and (4) jointly serve as the governing equations of the system. For the specific cases studied by the DFT simulations with the tube axis aligned along the $x$ direction, we obtain the solution from Eqs. (3) and (4) as $\varepsilon_{y}=\varepsilon_{y l}=C_{x} k_{x}^{\lambda}$. (See the complete mathematical formulations in the Supplemental Material [21].) 
In conclusion, we have demonstrated that bending deformations in a variety of 2D crystals can induce lateral strains, termed as the bending Poisson effect. The novel effect is dimension dependent, chemical constitution and bonding structure sensitive, and highly anisotropic. The bending Poisson ratio $\mu$, defined as the ratio of the lateral strain to the curvature $k$, scales with the curvature in a power law as $\mu=C k^{\lambda-1}$ in most situations. Moreover, a revised plate theory is established to incorporate the bending Poisson effect in $2 \mathrm{D}$ crystals into the continuum theory.

The work is supported by the 973 programs (No. 2013CB932604 and No. 2012CB933403), NSF of China (No. 91023026), Fundamental Research Funds for the Central Universities (No. NP2013309), and Jiangsu Innovation Program for Graduate Education (No. CXLX13_138). We thank Dr. Z. H. Zhang and Dr. Z.L. $\mathrm{Hu}$ for discussions.

*wlguo@nuaa.edu.cn

[1] S. D. Poisson, Traité de Mécanique (Chez Courcier, Paris, 1811), Vol. 2, 476.

[2] G. N. Greaves, A. L. Greer, R. S. Lakes, and T. Rouxel, Nat. Mater. 10, 823 (2011).

[3] T. Rouxel, J. Am. Ceram. Soc. 90, 3019 (2007).

[4] M. Q. Jiang and L. H. Dai, Philos. Mag. Lett. 90, 269 (2010).

[5] E. Kittinger, J. Tichý, and E. Bertagnolli, Phys. Rev. Lett. 47, 712 (1981).

[6] R. Lakes, Science 235, 1038 (1987).

[7] N. R. Keskar and J. R. Chelikowsky, Nature (London) 358, 222 (1992).

[8] F. Song, J. Zhou, X. Xu, Y. Xu, and Y. Bai, Phys. Rev. Lett. 100, 245502 (2008).

[9] D. Sánchez-Portal, E. Artacho, and J. M. Soler, Phys. Rev. B, 59, 12678 (1999).

[10] Y. Wei, B. Wang, J. Wu, R. Yang, and M. L. Dunn, Nano Lett. 13, 26 (2013).

[11] G. Kresse and J. Furthmüller, Phys. Rev. B 54, 11169 (1996).

[12] G. Kresse and J. Hafner, Phys. Rev. B 47, 558 (1993).

[13] P. E. Blöchl, Phys. Rev. B 50, 17953 (1994).

[14] G. Kresse and D. Joubert, Phys. Rev. B 59, 1758 (1999).
[15] J. P. Perdew, K. Burke, and M. Ernzerhof, Phys. Rev. Lett. 77, 3865 (1996).

[16] For one-dimensional periodic cells that contain up to 160 atoms, a dense $K$-point mesh $(1 \times 1 \times 13)$ is used for Brillouin zone sampling and a conjugate gradient method is applied to relax the geometry until interatomic forces are less than $0.005 \mathrm{eV} / \AA$.

[17] K. F. Mak, C. Lee, J. Hone, J. Shan, and T. F. Heinz, Phys. Rev. Lett. 105, 136805 (2010).

[18] B. Radisavljevic, A. Radenovic, J. Brivio, V. Giacometti, and A. Kis, Nat. Nanotechnol. 6, 147 (2011).

[19] U. K. Gautam, S. R. C. Vivekchand, A. Govindaraj, G. U. Kulkarni, N. R. Selvi, and C. N. R. Rao, J. Am. Chem. Soc. 127, 3658 (2005).

[20] K. He et al., Nat. Phys. 6, 584 (2010).

[21] See Supplemental Material at http://link.aps.org/ supplemental/10.1103/PhysRevLett.112.205502 for the deformation electron density of bent single-layer $\mathrm{MoS}_{2}$; the lateral strain in graphene and $\mathrm{h}-\mathrm{BN}$ as a function of curvature; bending-induced lateral strains in single-layered gallium selenide, hydrogenated graphene, and hexagonal aluminum nitride; and the complete mathematical formulations of the revised plate theory of large deflection.

[22] K. Jeon et al., ACS Nano 5, 1042 (2011).

[23] J. Zhou, Q, Wang, Q. Sun, and P. Jena, Phys. Rev. B 81, 085442 (2010).

[24] The electrostatic interaction experienced by the fluorine atoms in the tensile side is relatively weaker as the interatom distance is enlarged by bending.

[25] D. B. Zhang, E. Akatyeva, and T. Dumitrica, Phys. Rev. Lett. 106, 255503 (2011).

[26] D. C. Elias et al., Science 323, 610 (2009).

[27] H. Sahin, S. Cahangirov, M. Topsakal, E. Bekaroglu, E. Akturk, R. Senger, and S. Ciraci, Phys. Rev. B 80, 155453 (2009).

[28] The plate theory of large deflection was established by von Karman in 1910. L. Landau and E. M. Lifshitz, Theory of Elasticity (Pergamon, New York, 1986), 3rd ed.

[29] The coordinate axes $x, y$ are aligned along the two orthogonal in-plane crystal directions and the axis $z$ is along the out-of-plane direction. $\sigma_{x}\left(\sigma_{y}, \sigma_{x y}\right)$ is the stress and $\varepsilon_{x}\left(\varepsilon_{y}, \varepsilon_{x y}\right)$ is the strain on the middle plane of the crystals. $E$, $G$, and $\nu$ are the tensile modulus, shear modulus, and conventional Poisson ratio, respectively, and $C_{x}\left(C_{y}\right)$ is the coefficient of the power law. 